\title{
Abkürzungen (Editionen, Zeitschriften, Reihen, Nachschlagewerke)
}

\begin{tabular}{|c|c|}
\hline AASS & Acta Sanctorum \\
\hline $\mathrm{AB}$ & Analecta Bollandiana \\
\hline ACO & Acta conciliorum oecumenicorum \\
\hline $\mathrm{AE}$ & L’année épigraphique \\
\hline AHR & American Historical Review \\
\hline AJA & American Journal of Archaeology \\
\hline AJAH & American Journal of Ancient History \\
\hline $\mathrm{AJPh}$ & American Journal of Philology \\
\hline AJSLL & American Journal of Semitic Languages and Literatures \\
\hline AKG & Archiv für Kulturgeschichte \\
\hline AncSoc & Ancient Society \\
\hline ANRW & Aufstieg und Niedergang der römischen Welt \\
\hline AnTard & Antiquité tardive \\
\hline AntCl & L’antiquité classique \\
\hline $\mathrm{APF}$ & Archiv für Papyrusforschung \\
\hline BAR & British Archaeological Reports \\
\hline BASOR & Bulletin of the American Schools of Oriental Research \\
\hline BASP & Bulletin of the American Society of Papyrologists \\
\hline BBA & Berliner Byzantinistische Arbeiten \\
\hline BBS & Berliner Byzantinistische Studien \\
\hline BBKL & Biographisch-bibliographisches Kirchenlexikon \\
\hline $\mathrm{BCH}$ & Bulletin de correspondance héllenique \\
\hline $\mathrm{BF}$ & Byzantinische Forschungen \\
\hline BHAC & Bonner Historia-Augusta-Colloquium \\
\hline BHG & Bibliotheca Hagiographica Graeca \\
\hline BHL & Bibliotheca hagiographica Latina antiquae et mediae aetatis \\
\hline BJ & Bonner Jahrbücher \\
\hline BKV & Bibliothek der Kirchenväter \\
\hline BMGS & Byzantine and Modern Greek Studies \\
\hline $\mathrm{BS} / \mathrm{EB}$ & Byzantine Studies/Études byzantines \\
\hline BSOAS & Bulletin of the School of Oriental and African Studies \\
\hline BSOS & Bulletin of the School of Oriental Studies \\
\hline $\mathrm{BSl}$ & Byzantinoslavica \\
\hline Byz & Byzantion \\
\hline ByzBulg & Byzantinobulgarica \\
\hline $\mathrm{BZ}$ & Byzantinische Zeitschrift \\
\hline C. & Codex Iustinianus, ed. P. Krueger \\
\hline $\mathrm{CAH}$ & The Cambridge Ancient History \\
\hline CahArch & Cahiers archéologiques \\
\hline
\end{tabular}


CAVT

CC

CCSG

CCSL

CCCM

CFHB

CIG

CIL

CJ

CPG

$\mathrm{CPh}$

CPL

CPPM

CQ

CR

CRAI

CSHB

CSCO

CSEL

CTh

D.

DA

DACL

DHGE

DNP

DOP

DOS

DOT

EHR

FGrHist

FHG

FM

FMSt

GCS

GRBS

Gym

Hell

HdAW

$\mathrm{HSPh}$

HThR
Clavis apocryphorum Veteris Testamenti

Corpus christianorum

Corpus christianorum, series Graeca

Corpus christianorum, series Latina

Corpus Christianorum continuatio medievalis

Corpus fontium historiae byzantinae

Corpus Inscriptionum Graecarum

Corpus Inscriptionum Latinarum

Classical Journal

Clavis patrum Graecorum

Classical Philology

Clavis patrum Latinorum

Clavis patristica pseudepigraphorum medii aevi

Classical Quarterly

Classical Review

Comptes rendus des séances de l'Académie des inscriptions et belles-lettres

Corpus scriptorum historiae Byzantinae

Corpus scriptorum christianorum Orientalium

Corpus scriptorum ecclesiasticorum Latinorum

Codex Theodosianus

Digesta, ed. Th. Mommsen

Deutsches Archiv für Erforschung des Mittelalters

Dictionnaire d'archéologie chrétienne et de liturgie

Dictionnaire d'histoire et de géographie ecclésiastiques

Der neue Pauly

Dumbarton Oaks Papers

Dumbarton Oaks Studies

Dumbarton Oaks Texts

English Historical Review

Felix Jacoby (Hg.): Die Fragmente der griechischen Historiker. Leiden 1923-1958.

Fragmenta historicorum Graecorum, collegit, disposuit, notis et prolegomenis illustravit C. Mullerus, I-VI. Paris 1841/1870.

Fontes Minores

Frühmittelalterliche Studien

Die griechischen christlichen Schriftsteller

Greek, Roman and Byzantine Studies

Gymnasium

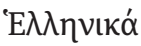

Handbuch der Altertumswissenschaft

Harvard Studies in Philology

Harvard Theological Review 


\begin{tabular}{|c|c|}
\hline $\mathrm{HZ}$ & Historische Zeitschrift \\
\hline I. & Institutiones, ed. P. Krueger \\
\hline IG & Inscriptiones Graecae \\
\hline ILS & Inscriptiones Latinae Selectae \\
\hline IstMitt & Istanbuler Mitteilungen \\
\hline JAOS & Journal of the American Oriental Society \\
\hline $\mathrm{JbAC}$ & Jahrbuch für Antike und Christentum \\
\hline JDAI & Jahrbuch des Deutschen Archäologischen Institutes \\
\hline JECS & Journal of Early Christian Studies \\
\hline JEH & Journal of Ecclesiastical History \\
\hline JHS & Journal of Hellenic Studies \\
\hline JÖAI & Jahrbuch des Österreichischen Archäologischen Instituts \\
\hline JÖB & Jahrbuch der Österreichischen Byzantinistik \\
\hline JÖBG & Jahrbuch der Österreichischen Byzantinischen Gesellschaft \\
\hline JRA & Journal of Roman Archaeology \\
\hline JRGZM & Jahrbuch des Römisch-Germanischen Zentralmuseums \\
\hline JRS & Journal of Roman Studies \\
\hline JS & Journal des Savants \\
\hline JThS & Journal of Theological Studies \\
\hline LACL & $\begin{array}{l}\text { Lexikon der antiken christlichen Literatur, hg. von Siegmar } \\
\text { Döpp und Wilhelm Geerlings. Freiburg/Basel/Wien }{ }^{3} 2002 .\end{array}$ \\
\hline LAW & Lexikon der Alten Welt \\
\hline LexMa & Lexikon des Mittelalters \\
\hline LIMC & Lexicon Iconographicum Mythologiae Classicae \\
\hline LThK & Lexikon für Theologie und Kirche \\
\hline $\operatorname{MDAI}(\mathrm{A})$ & $\begin{array}{l}\text { Mitteilungen des Deutschen Archäologischen Instituts, Athe- } \\
\text { nische Abteilung }\end{array}$ \\
\hline MDAI(R) & $\begin{array}{l}\text { Mitteilungen des Deutschen Archäologischen Instituts, Römi- } \\
\text { sche Abteilung }\end{array}$ \\
\hline MEFRA & Mélanges de l’École française de Rome: Antiquité \\
\hline MEFRM & $\begin{array}{l}\text { Mélanges de l’École française de Rome: Moyen âge - Temps } \\
\text { modernes }\end{array}$ \\
\hline MGH & Monumenta Germaniae Historica \\
\hline AA & Auctores antiquissimi \\
\hline Capit. & Capitularia \\
\hline Conc. & Concilia \\
\hline Epp. & Epistolae \\
\hline Poet. & Poetae Latini aevi Carolini \\
\hline SS & Scriptores \\
\hline SS rer. Lang. et It. & Scriptores rerum Langobardicarum et Italicarum \\
\hline SS rer. Merov. & Scriptores rerum Merovingicarum \\
\hline $\mathrm{MH}$ & Museum Helveticum \\
\hline
\end{tabular}


MIÖG

Mus

N.

PBE

PBSR

$\mathrm{PCPhS}$

PG

PIR

PL

PLRE

PmbZ

PO

P\&P

RA

RAC

$\mathrm{RB}$

$\mathrm{RbK}$

RE

REA

REArm

REAug

REB

REG

REL

RGA

RGG

$\mathrm{RH}$

RHE

RhM

RHR

$\mathrm{RPh}$

RQ

SC

SEG

SO

Spec

StP

StT

SubHag

TAM
Mitteilungen des Instituts für Österreichische Geschichtsforschung

Le Muséon

Novellae, edd. R. Schöll/W. Kroll

Prosopography of the Byzantine Empire

Papers of the British School at Rome

Proceedings of the Cambridge Philological Society

Patrologia Graeca

Prosopographia Imperii Romani

Patrologia Latina

Prosopography of the Later Roman Empire

Prosopographie der mittelbyzantinischen Zeit

Patrologia Orientalis

Past and Present

Revue archéologique

Reallexikon für Antike und Christentum

Revue bénédictine

Reallexikon zur byzantinischen Kunst

Pauly's Real-Encyclopaedie der classischen Altertumswissenschaft

Revue des études anciennes

Revue des études arméniennes

Revue des études augustiniennes

Revue des études byzantines

Revue des études grecques

Revue des études latines

Reallexikon der germanischen Altertumskunde

Religion in Geschichte und Gegenwart

Revue historique

Revue d'histoire ecclésiastique

Rheinisches Museum für Philologie

Revue de l'histoire des religions

Revue philologique

Römische Quartalschrift für christliche Altertumskunde und Kirchengeschichte

Sources chrétiennes

Supplementum epigraphicum Graecum

Symbolae Osloenses

Speculum

Studia Patristica

Studi e testi

Subsidia Hagiographica

Tituli Asiae Minoris 
TAPA

ThLL

ThLZ

ThQ

ThR

TIB

TRE

TRW

TU

VChr

VetChr

$\mathrm{VuF}$

WBS

WdF

WSt

WZKM

ZAC

ZKG

ZPE

ZRG germ. Abt.

ZRG kan. Abt.

ZRG rom. Abt.

ZThK
Transactions and Proceedings of the American Philological Association

Thesaurus Linguae Latinae

Theologische Literaturzeitung

Theologische Quartalschrift

Theologische Rundschau

Tabula Imperii Byzantini

Theologische Realenzyklopädie

Transformation of the Roman World

Texte und Untersuchungen zur Geschichte der altchristlichen

Literatur

Vigiliae Christianae

Vetera Christianorum

Vorträge und Forschungen

Wiener Byzantinistische Studien

Wege der Forschung

Wiener Studien

Wiener Zeitschrift für die Kunde des Morgenlandes

Zeitschrift für antikes Christentum

Zeitschrift für Kirchengeschichte

Zeitschrift für Papyrologie und Epigraphik

Zeitschrift der Savigny-Stiftung für Rechtsgeschichte, germanistische Abteilung

Zeitschrift der Savigny-Stiftung für Rechtsgeschichte, kanonistische Abteilung

Zeitschrift der Savigny-Stiftung für Rechtsgeschichte, romanistische Abteilung

Zeitschrift für Theologie und Kirche 
